\title{
Antimycobacterial activity of DNA intercalator inhibitors of Mycobacterium tuberculosis primase DnaG
}

\author{
Chathurada Gajadeera' ${ }^{1,4}$, Melisa J Willby ${ }^{2,4}$, Keith D Green ${ }^{1}$, Pazit Shaul ${ }^{3}$, Micha Fridman ${ }^{3}$, \\ Sylvie Garneau-Tsodikova ${ }^{1}$, James E Posey ${ }^{2}$ and Oleg V Tsodikov ${ }^{1}$
}

Owing to the rise in drug resistance in tuberculosis combined with the global spread of its causative pathogen, Mycobacterium tuberculosis (Mtb), innovative anti mycobacterial agents are urgently needed. Recently, we developed a novel primasepyrophosphatase assay and used it to discover inhibitors of an essential Mtb enzyme, primase DnaG (Mtb DnaG), a promising and unexplored potential target for novel antituberculosis chemotherapeutics. Doxorubicin, an anthracycline antibiotic used as an anticancer drug, was found to be a potent inhibitor of $M t b$ DnaG. In this study, we investigated both inhibition of $M$ tb DnaG and the inhibitory activity against in vitro growth of $M t b$ and $M$. smegmatis $(M s m)$ by other anthracyclines, daunorubicin and idarubicin, as well as by less cytotoxic DNA intercalators: aloe-emodin, rhein and a mitoxantrone derivative. Generally, low- $\mu \mathrm{M}$ inhibition of $M t b$ DnaG by the anthracyclines was correlated with their low- $\mu \mathrm{M}$ minimum inhibitory concentrations. Aloe-emodin displayed threefold weaker potency than doxorubicin against $M t b$ DnaG and similar inhibition of $\mathbf{M s m}$ (but not $M$ tb) in the mid$\mu \mathrm{M}$ range, whereas rhein (a close analog of aloe-emodin) and a di-glucosylated mitoxantrone derivative did not show significant inhibition of $M t b$ DnaG or antimycobacterial activity. Taken together, these observations strongly suggest that several clinically used anthracyclines and aloe-emodin target mycobacterial primase, setting the stage for a more extensive exploration of this enzyme as an antibacterial target.

The Journal of Antibiotics (2015) 68, 153-157; doi:10.1038/ja.2014.131; published online 24 September 2014

\section{INTRODUCTION}

Global spread of multidrug-resistant M. tuberculosis ( $M t b)$ strains and emergence of extensively drug-resistant tuberculosis (TB) call for innovative approaches for treating TB. As conventional first- and second-line anti-TB drugs are gradually rendered less useful due to drug resistance, ${ }^{1}$ and the number of drug candidates in the anti-TB pipeline is low, ${ }^{2}$ novel targets and agents inhibiting them need to be explored. Bacterial primase DnaG, a DNA-dependent RNA polymerase that synthesizes oligonucleotide primers during DNA replication, has been known for several decades to be essential for both chromosomal DNA replication and cell division. ${ }^{3-6}$ DnaG is conserved in bacteria and is distinct from eukaryotic and archaeal primases. ${ }^{7}$ In addition, the catalytic TOPRIM (topoisomerase-primase) domain of DnaG shares a structural fold with DNA gyrase, a target of the clinically useful fluoroquinolone antibiotics. ${ }^{8}$ Therefore, DnaG is an attractive potential target for discovery and development of novel antibacterial agents. The requirements of radioactivity as a detection method and accessory DNA replication factors to boost primase activity in traditional in vitro primase activity assays have impeded facile identification and characterization of DnaG inhibitors, and, until recently, no potent (low- $\mu \mathrm{M}$ or better) inhibitors of DnaG were reported.

We recently developed a coupled colorimetric primase-pyrophosphatase assay for measurement of DnaG activity and applied this assay in a high-throughput screening (HTS) of small-molecule libraries to identify inhibitors of DnaG and another essential enzyme, inorganic pyrophosphatase (PPiase). ${ }^{9}$ HTS applications of this assay to Mtb DnaG $^{9}$ and Bacillus anthracis $\mathrm{DnaG}^{10}$ yielded low- $\mu \mathrm{m}$ inhibitors of these enzymes. Doxorubicin, an anthracycline antibiotic of bacterial origin used in clinics as an anticancer drug, was identified in these studies as a potent inhibitor of both DnaG enzymes. The cytotoxic activity of doxorubicin against cancer cells originates from its inhibition of topoisomerase II, ${ }^{11}$ a eukaryotic homolog of gyrase, by creating a ternary complex with topoisomerase II and dsDNA. Even though the antibacterial mechanism of action of doxorubicin has not been extensively investigated, early studies with this compound reported it

${ }^{1}$ Department of Pharmaceutical Sciences, University of Kentucky, Lexington, KY, USA; ${ }^{2}$ Division of Tuberculosis Elimination, National Center for HIV/AIDS, Viral Hepatitis, STD, and TB Prevention, Centers for Disease Control and Prevention, Atlanta, GA, USA and ${ }^{3}$ School of Chemistry, Tel Aviv University, Tel Aviv, Israel

${ }^{4}$ These authors contributed equally to this work.

Correspondence: Dr S Garneau-Tsodikova or Dr OV Tsodikov, Department of Pharmaceutical Sciences, University of Kentucky, BioPharm Complex, Room 423, 789 South Limestone Street, Lexington, KY 40536-0596, USA or Dr JE Posey, Division of Tuberculosis Elimination, National Center for HIV/AIDS, Viral Hepatitis, STD, and TB Prevention, Centers for Disease Control and Prevention, Atlanta, GA 30333, USA.

E-mail: sylviegtsodikova@uky.edu or oleg.tsodikov@uky.edu or hzp9@cdc.gov

Received 18 June 2014; revised 29 July 2014; accepted 13 August 2014; published online 24 September 2014 
as a DNA replication inhibitor, ${ }^{12}$ whereas its inhibition of gyrase was shown to be too weak to explain its antibacterial potency. ${ }^{13}$ Our recent findings, taken together with these earlier observations, suggest that doxorubicin inhibits bacterial cell growth by inhibiting DnaG.

In our search for other potentially therapeutically useful inhibitors of DnaG, we explored several anthracycline-based DNA intercalators as well as less toxic natural anthranoids. We investigated the inhibitory potency of these agents against the activity of purified $M t b$ DnaG, as well as measured their minimum inhibitory concentrations (MICs) in the in vitro cultures of $M t b$ strain $\mathrm{H} 37 \mathrm{Rv}$ and M. smegmatis str. $\mathrm{mc}^{2}$ 155 (Msm), a fast-growing Mycobacterium model. Msm and Mtb DnaGs are nearly identical ( $82 \%$ sequence identity), with most differences exhibited in the C-terminal, replicative helicase-binding domain, which is not required for the primer synthesis activity of DnaG in vitro. ${ }^{8}$ A correlation between the in vitro antagonism of $M t b$ DnaG and the inhibition of the mycobacterial cell growth for these compounds strongly suggests that DnaG inhibition contributes significantly to their antimicrobial activity.

\section{MATERIALS AND METHODS}

\section{Expression and purification of $M t b$ DnaG}

The $M t b$ DnaG protein was expressed and purified by a modified version of our previously published protocol, ${ }^{9}$ as follows. Protein expression was carried out in Escherichia coli BL21 (DE3) cells cultured in LB broth supplemented with ampicillin $\left(100 \mu \mathrm{g} \mathrm{ml}^{-1}\right)$. A $2-1$ culture was grown to an attenuance at $600 \mathrm{~nm}$ of 0.2 and induced with $0.5 \mathrm{~mm}$ of IPTG and incubated for $16 \mathrm{~h}$ at $18^{\circ} \mathrm{C}$. (Note: All purification steps were carried out at $4^{\circ} \mathrm{C}$, without freezing the bacterial pellet). The cells were harvested and the pellet was suspended in $50 \mathrm{ml}$ of lysis buffer ( $40 \mathrm{~mm}$ Tris pH 8.0, $600 \mathrm{~mm} \mathrm{NaCl}, 5 \%$ v/v glycerol, $1 \mathrm{~mm}$ PMSF, 2 mм $\mathrm{MgCl}_{2}$ and $2 \mathrm{~mm} \beta$-mercaptoethanol). The cells were disrupted by sonication on ice and clarified by centrifugation at $40000 \times g$ for $40 \mathrm{~min}$. The supernatant was filtered through a $0.45-\mu \mathrm{m}$ Millex-HV PVDF filter (Millipore, Billerica, MA, USA) and applied to a 1-ml Ni-IMAC HisTrap FF column (GE Healthcare Life Sciences, Pittsburgh, PA, USA) equilibrated with lysis buffer. The column was washed with $20 \mathrm{ml}$ of lysis buffer containing $50 \mathrm{~mm}$ imidazole, and the protein was eluted with $11 \mathrm{ml}$ of lysis buffer containing $500 \mathrm{~mm}$ imidazole. The fractions containing protein were loaded onto a size-exclusion S-200 column (GE Healthcare Life Sciences) equilibrated in gel filtration buffer ( $40 \mathrm{~mm}$ Tris pH 8.0, $600 \mathrm{~mm} \mathrm{NaCl}, 5 \% \mathrm{v} / \mathrm{v}$ glycerol and $2 \mathrm{~mm}$ of $\beta$-mercaptoethanol), and the protein-containing fractions were pooled and concentrated using an Amicon Ultra-15 centrifugal filter device (Millipore) to $3 \mathrm{mg} \mathrm{ml}^{-1}$ final concentration. The protein was then flash frozen in $30-\mu \mathrm{l}$ aliquots in liquid nitrogen and stored at $-80^{\circ} \mathrm{C}$. The freezing process did not affect the protein activity; the aliquots were used immediately upon thawing and were not reused later or refrozen. The presence of the N-terminal His-tag did not have any effect on the protein activity, as compared with the previously published results. ${ }^{9} M t b$ PPiase was expressed and purified as previously described. ${ }^{9}$

\section{Dose-response assays}

The dose-response assays were performed in 96-well plates as previously described. ${ }^{9}$ Primase activity measurements were performed in triplicate. The dose-response curves were analyzed by nonlinear regression with SigmaPlot 9.0 (SysStat Software, San Jose, CA, USA). The following general dose-response equation was used in nonlinear regression data fitting of the relative protein activity, $f$, as a function of inhibitor concentration, [I], with $\mathrm{IC}_{50}$ and Hill coefficient, $n$, as fitting parameters:

$$
f=\frac{1}{1+\left(\frac{[\mathrm{II}]}{\mathrm{IC}_{50}}\right)^{n}}
$$

Hill coefficients greater than unity were observed for the anthracyclines and the mitoxantrone derivative, an indication of multiple molecules of these inhibitors interacting with DNA/DNA-DnaG complex or self-association of these compounds, likely through ring-ring stacking interactions. Inhibition of $M t b$ PPiase alone by all the compounds of interest was tested by an analogous assay with $50 \mu \mathrm{m}$ of sodium pyrophosphate as a substrate, in the absence of $\mathrm{Mtb}$ DnaG, DNA and NTP. Absorbance was measured at $30 \mathrm{~s}$ (when approximately half of the $\mathrm{PP}_{\mathrm{i}}$ was cleaved), and at $4 \mathrm{~min}$. The reactions were carried out without a compound and at $100 \mu \mathrm{m}$ of each compound. No inhibition of $\mathrm{Mtb}$ PPiase was observed by any of the molecules tested.

Determination of MIC values against mycobacterial culture growth Determination of MIC values against Mtb by the alamar blue assay. The MIC for each compound was determined using the microplate alamar blue assay as previously described with slight modifications. ${ }^{14}$ All compounds were diluted to $20 \mu \mathrm{M}$ working stocks in $7 \mathrm{H} 9$ medium. The working stocks were twofold serially diluted to achieve compound concentrations between 640 and $0.156 \mu \mathrm{M}$. A volume of $200 \mu \mathrm{l}$ sterile distilled water $\left(\mathrm{ddH}_{2} \mathrm{O}\right)$ was added to all perimeter wells of 96-well test plates to reduce evaporation from test wells. The 7H9 medium without compound served as a growth control and uninoculated 7H9 medium was included as a sterility control. A volume of $100 \mu \mathrm{l} 7 \mathrm{H} 9$ medium containing test compounds at the various concentrations was added to the other wells. Mtb strain $\mathrm{H} 37 \mathrm{Rv}$ was inoculated from frozen stocks into Middlebrook 7H9 broth supplemented with 10\% albumin-dextrose-catalase (ADC, BD Biosciences, San Jose, CA, USA), 0.05\% Tween 80 (Sigma-Aldrich, St Louis, MO, USA) and $0.4 \% \mathrm{v} / \mathrm{v}$ glycerol and incubated at $37^{\circ} \mathrm{C}$ until turbid. Cultures were then diluted to an attenuance at $600 \mathrm{~nm}$ of 0.2 in fresh $7 \mathrm{H} 9$ medium, then additionally diluted $1: 25 \mathrm{in} 7 \mathrm{H} 9$, added to $50 \mathrm{ml}$ polypropylene tubes containing glass beads, vortexed for $30 \mathrm{~s}$, allowed to settle for $10 \mathrm{~min}$ and $100 \mu \mathrm{l}$ was distributed into the wells of columns $2-10$ of the test plate making the final concentration of test compounds $0.078-10 \mu \mathrm{M}$. Compounds with initial MICs $>10 \mu \mathrm{M}$ were similarly tested at compound concentrations between 10 and $320 \mu \mathrm{m}$. Compound screening was carried out on biological replicates in triplicate. After the plates were incubated at $37^{\circ} \mathrm{C}$ in a humid environment for 5-6 days, $40 \mu \mathrm{l}$ of alamar blue diluted $1: 2$ in $10 \%$ Tween 80 was added to each well and the plates were incubated at $37^{\circ} \mathrm{C}$. The color of each well was preliminarily evaluated $24 \mathrm{~h}$ after the addition of alamar blue, with a final evaluation after $48 \mathrm{~h}$. Alamar blue changes from indigo blue to pink as a result of bacterial growth. The lowest concentration of compound that resulted in no color change was recorded as the MIC for each compound.

Determination of MIC values against Msm. MIC values were determined using the double-dilution method starting at $150 \mu \mathrm{g} \mathrm{ml}^{-1}$ in a total volume of $200 \mu \mathrm{l}$. Dilutions of a Msm str. $\mathrm{mc}^{2} 155$ culture were added to the solutions of the tested compounds dissolved in Mueller-Hinton broth. Bacteria were grown at $37^{\circ} \mathrm{C}$ until cultures became turbid, 2 days. MIC values were determined as the last dilution to have no bacterial growth as determined by alamar blue (resazurin; $5 \mu \mathrm{l}$ of a $7 \mathrm{mg} \mathrm{ml}^{-1}$ solution) staining for 2 days. Wells showing no color change from the standard blue color were determined to have no bacterial growth.

\section{RESULTS}

\section{Inhibition of $M t b$ DnaG by a series of DNA intercalator agents}

By using our recently developed colorimetric coupled primasepyrophosphatase assay with purified $M t b$ DnaG and $M t b$ PPiase, we investigated inhibition of purified $M t b$ primase DnaG by seven compounds: the anthracyclines doxorubicin, daunorubicin and idarubicin, the natural anthranoids aloe-emodin and rhein, an anthracenedione di-glucosyl mitoxantrone and the fluoroquinolone ofloxacin (Figure 1). These compounds are structurally diverse DNA intercalating agents, previously reported to have some antibacterial activity. Consistent with previously observed low- $\mu \mathrm{m}$ inhibition of $M t b$ DnaG by doxorubicin, ${ }^{9}$ structurally related daunorubicin and idarubicin also displayed $\mathrm{IC}_{50}$ values for $M t b$ DnaG inhibition in the low- $\mu \mathrm{M}$ range: $\mathrm{IC}_{50}=7.2 \pm 0.3$ and $8.2 \pm 1.1 \mu \mathrm{M}$ for daunorubicin and idarubicin, respectively (Figure $2 \mathrm{a}$ and Table 1). Aloe-emodin, a plant-derived compound with cathartic and modest anticancer activities, ${ }^{15}$ exhibited significant, albeit weaker inhibition of this enzyme with $\mathrm{IC}_{50}=19 \pm 2$ $\mu \mathrm{M}$ (Figure $2 \mathrm{~b}$ and Table 1 ). A close structural analog of aloe-emodin, also with cathartic and anticancer activities, rhein, ${ }^{16}$ however, did not 

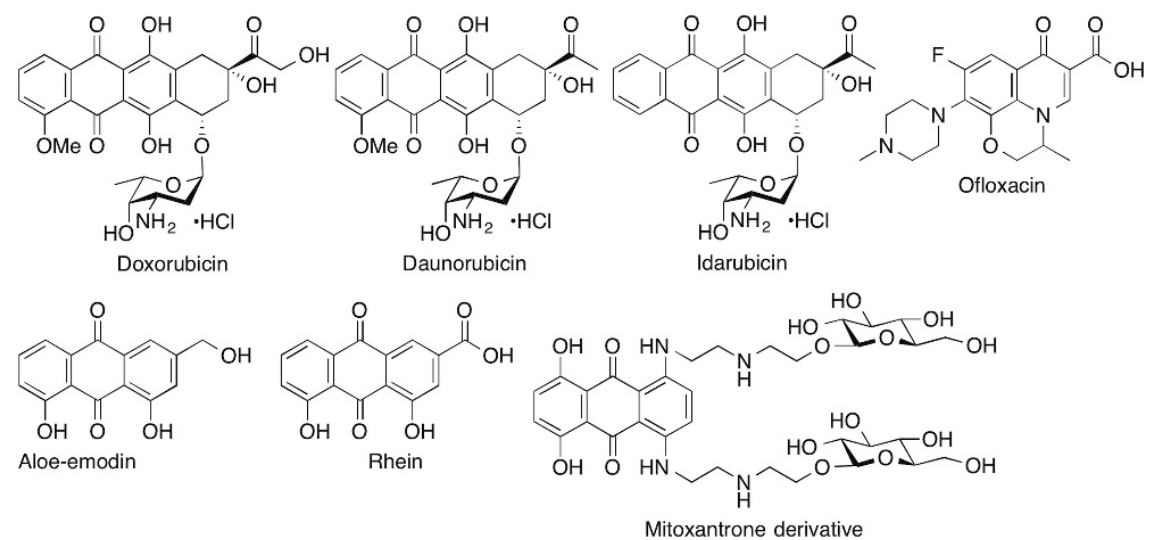

Mitoxantrone derivative

Figure 1 Structures of small molecules used in this study.

a

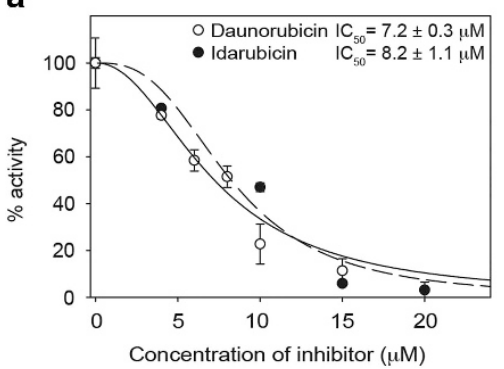

b

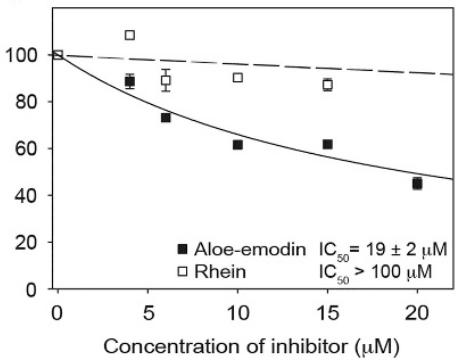

C

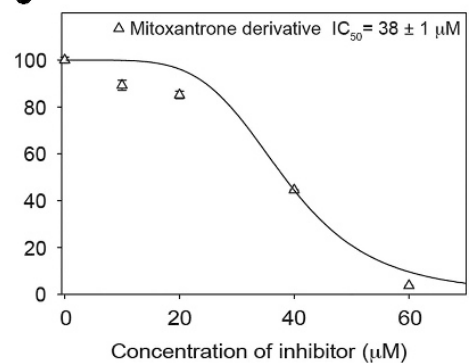

Figure 2 Primase-PPiase dose-response assays with (a) daunorubicin (white circles) and idarubicin (black circles), (b) aloe-emodin (black squares) and rhein (white squares) and (c) a glycosylated mitoxantrone derivative (white triangles). The assays were performed with $1.25 \mu \mathrm{m}$ of DNA, $110 \mu \mathrm{m}$ of NTP and $0.6 \mu \mathrm{m}$ of $\mathrm{Mtb}$ DnaG. Daunorubicin $\left(\mathrm{IC}_{50}=7.2 \pm 0.3 \mu \mathrm{M}\right)$ and idarubicin $\left(\mathrm{IC}_{50}=8.2 \pm 1.1 \mu \mathrm{m}\right)$ showed low- $\mu \mathrm{m}$ range inhibition. Daunorubicin and idarubicin could be well modeled by using the Hill coefficients $(n)$ of $2.1 \pm 0.1$ and $2.8 \pm 0.8$, respectively. Aloe-emodin displayed weaker inhibition of the enzyme $\left(\mathrm{IC}_{50}=19 \pm 2 \mu \mathrm{m}\right)$, and rhein did not show any significant inhibition $\left(\mathrm{IC}_{50}>100 \mu \mathrm{m}\right)$. The mitoxantrone derivative showed much weaker inhibition $\left(\mathrm{IC}_{50}=38 \pm 1 \mu \mathrm{M}\right)$ with $n=5.0 \pm 1.8$.

Table 1 Mtb DnaG and $M$ tb PPiase $\mathrm{IC}_{50}$ values and mycobacterial MIC values for DNA intercalators tested

\begin{tabular}{lcccc}
\hline Compound & $\begin{array}{c}\text { Mtb DnaG } \\
I C_{50}(\mu \mathrm{M})\end{array}$ & $\begin{array}{c}\text { Mtb PPiase } \\
I C_{50}(\mu \mathrm{M})\end{array}$ & $\begin{array}{c}\text { Msm MIC } \\
(\mu \mathrm{M})\end{array}$ & $\begin{array}{c}\text { Mtb MIC } \\
(\mu \mathrm{M})\end{array}$ \\
\hline Doxorubicin & $7.7 \pm 0.5^{\mathrm{a}}$ & $>100$ & 8 & 5 \\
Daunorubicin & $7.2 \pm 0.3$ & $>100$ & 0.5 & 1.25 \\
Idarubicin & $8.2 \pm 1.1$ & $>100$ & 0.6 & 80 \\
Aloe-emodin & $19 \pm 2$ & $>100$ & 70 & $>320$ \\
Rhein & $>100$ & $>100$ & $>260$ & $>320$ \\
Mitoxantrone derivative & $38 \pm 1$ & $>100$ & $>100$ & $>320$ \\
Ofloxacin & $>100$ & $>100$ & $3^{\mathrm{b}}$ & $3^{\mathrm{c}}$ \\
\hline
\end{tabular}

Abbreviations: MIC, minimum inhibitory concentration; Msm, Mycobacterium smegmatis; Mtb, M. tuberculosis; PPiase, inorganic pyrophosphatase.

aThis $\mathrm{IC}_{50}$ value was previously reported. ${ }^{9}$

bThis MIC value was previously reported. ${ }^{26}$

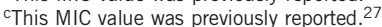

inhibit $M t b$ DnaG to a measurable degree $\left(\mathrm{IC}_{50}>100 \mu \mathrm{M}\right)$ (Figure $2 \mathrm{~b}$ and Table 1). Another DNA intercalator structurally distinct from any of the above compounds, a derivative of an anticancer drug, mitoxantrone, inhibited $M t b$ DnaG about twofold more weakly $\left(\mathrm{IC}_{50}=38 \pm 1 \mu \mathrm{M}\right)$ than aloe-emodin (Figure $2 \mathrm{c}$ and Table 1). Finally, the fluoroquinolone ofloxacin, which targets DNA gyrase, did not show any observable inhibition of Mtb DnaG (Table 1).

\section{Inhibition of in vitro growth of $M t b$ and $M s m$ by the DNA} intercalators

To test whether the identified $M t b$ DnaG inhibitors halt growth of mycobacteria, and not only function on purified enzyme, we determined the MIC values for all seven molecules studied against two strains of mycobacteria, Msm $\mathrm{mc}^{2} 155$ and Mtb H37Rv (Table 1). Doxorubicin inhibited $M s m$ and $M t b$ with an MIC of 8 and $5 \mu \mathrm{M}$, respectively, comparable with the $\mathrm{IC}_{50}$ values in the primase assay. Daunorubicin displayed more potent growth inhibition of $\mathrm{Msm}$ and $M t b$, with an MIC of 0.5 and $1.25 \mu \mathrm{M}$, respectively. On the other hand, idarubicin strongly inhibited $\mathrm{Msm}(\mathrm{MIC}=0.6 \mu \mathrm{M})$, but not $\mathrm{Mtb}$ $(\mathrm{MIC}=80 \mu \mathrm{M})$. Aloe-emodin displayed intermediate potency against $M s m(\mathrm{MIC}=70 \mu \mathrm{M})$ and did not show inhibition of $M t b$ up to $320 \mu \mathrm{M}$. Interestingly, rhein did not show any inhibition of either Msm $(\mathrm{MIC}>260 \mu \mathrm{M})$ or $\mathrm{Mtb} \quad(\mathrm{MIC}>320 \mu \mathrm{M})$. Similarly, the diglucosylated mitoxantrone derivative did not display inhibition of either Msm $(\mathrm{MIC}>100 \mu \mathrm{M})$ or $M t b(\mathrm{MIC}>320 \mu \mathrm{M})$.

\section{DISCUSSION}

Primase DnaG has recently emerged as a promising potential target for the discovery of antibacterial agents., ${ }^{9,17}$ HTS-driven search for inhibitors of $M t b$ DnaG and Bacillus anthracis DnaG yielded doxorubicin, a Streptomyces natural product and a known bacterial DNA replication inhibitor ${ }^{12}$ that is a highly efficient antineoplastic agent commonly used in the treatment of hematopoietic and solid tumors. These studies strongly suggest that doxorubicin evolved to target 
DnaG and that anthracyclines in general and other DNA intercalators may have antimycobacterial activity and efficiently inhibit DnaG. Indeed, we showed here that the anthracycline daunorubicin, like doxorubicin, is an inhibitor of DnaG with similar potent antibacterial activity against both $M s m$ and $M t b$. In fact, daunorubicin is 4 to 16-fold more potent than doxorubicin in growth inhibition of both mycobacteria. This may be attributed to an additional mode of action such as oxidative damage induced by this anthracycline, more efficient penetration through the cell envelope or more potent inhibition of DnaG in the context of the entire replication machinery. On the other hand, idarubicin, similar to daunorubicin, is more potent than doxorubicin against $M s m$, but it appears to be less potent than doxorubicin in inhibiting $M t b$. Specific structural features of the cell envelope of $M t b$ may prevent idarubicin from penetrating into the cytoplasm of $M t b$.

Another DNA intercalator of a different structural class, aloeemodin, displayed MIC values against $\mathrm{Msm}$ in a mid- $\mu \mathrm{M}$ range, but exhibited no growth inhibition of $M t b$ strain H37Rv. A recent study showed that aloe-emodin is a weak $(\sim 100 \mu \mathrm{M})$ inhibitor of H37Ra strain of $M t b .{ }^{18}$ The weaker activity of aloe-emodin against $M t b$ than against Msm may be explained by differences in the cell envelopes of these two mycobacteria. It has been shown that the presence of surface-exposed C-type glycopeptidolipids (GPLs) in Msm increases cell-wall barrier permeability. These GPLs are species-specific and found only in nontuberculous mycobacterial species. The GPLdeficient mutant strain exhibited an increase of the cell hydrophobicity. ${ }^{19}$ The many polar groups of emodin and its small size may enable it to cross the outer membrane of Msm more efficiently. This phenomenon is thought to account for the resistance of the outer membrane of Msm to rifampicin, a hydrophobic antibiotic. ${ }^{20}$ Furthermore, the presence of porins, mainly MspA, in the outer membrane of Msm may allow the transport of small molecules decorated with hydrophilic groups. A mutant strain with a deletion of $m s p A$ exhibited a ninefold and fourfold reduction in permeability for cephaloridine and glucose, respectively. ${ }^{21}$ No sequence homologs to MspA were identified in $M t b$.

In contrast, a close analog of aloe-emodin, rhein (US patent US5652265, 1997), which contains a carboxylic acid moiety instead of hydroxymethyl groups of aloe-emodin, shows no measurable growth inhibition of either $\mathrm{Msm}$ or $\mathrm{Mtb}$. In semiquantitative agreement with these MIC measurements, aloe-emodin is a mid- $\mu \mathrm{M}$ range inhibitor of $M t b$ DnaG, whereas rhein does not inhibit this enzyme to an observable extent. These data strongly suggest that inhibition of DnaG has a major role in the antibacterial activity of aloe-emodin. An analog of aloe-emodin, emodin, and rhein appear to cross a variety of bacterial cell envelopes of nonmycobacteria equally well, ${ }^{22}$ although we cannot exclude the possibility that the inactivity of the charged rhein against both mycobacteria may be due to some specific features of the mycobacterial envelope, such as multidrug resistance pumps. ${ }^{23}$ A recent study demonstrated that emodin is an $\sim 100 \mu \mathrm{M}$ inhibitor of gyrase (whose catalytic domain shares the catalytic TOPRIM fold with DnaG) and topoisomerase $I^{8}{ }^{8}$ which, together with our results, suggests that the emodin scaffold is efficient in targeting gyrases and bacterial primases. In the same study, halogenated emodins or haloemodins were shown to inhibit bacterial topoisomerases much more potently than the parent compound. ${ }^{24}$ Extending the correlation of MIC and $\mathrm{IC}_{50}$ of DnaG inhibition among the DNA intercalators tested here, the glycosylated derivative of mitoxantrone was inert toward $M s m$ and $M t b$ and it was very weakly inhibitory to $M t b$ DnaG $\left(\mathrm{IC}_{50}=38 \pm 1 \mu \mathrm{M}\right)$. Notably, a recent study identifies mitoxantrone as an inhibitor of $E$. coli gyrase $\left(\mathrm{IC}_{50}=80 \mu \mathrm{M}\right) .{ }^{25}$ Therefore, mitoxantrones appear to exhibit an analogous target profile to that of emodins. Another DNA intercalator of a distinct structural class, the fluoroquinolone ofloxacin, a known potent antimycobacterial agent $\left(\mathrm{MIC}=3 \mu \mathrm{M}\right.$ against both $\mathrm{Msm}^{26}$ and $M t b^{27}$ ) acting as an inhibitor of another DNA-binding enzyme, gyrase, did not have an observable inhibition effect on $\mathrm{Mtb}$ DnaG $\left(\mathrm{IC}_{50}>100 \mu \mathrm{M}\right)$, serving as a control in this study.

The mechanism of action of DNA-binding antibiotics in bacteria has not been well understood, except for (i) agents that result in DNA breaks indirectly through binding to DNA breaking enzyme-DNA complexes, such as quinolones, and (ii) agents that directly result in DNA breaks, such as bleomycin or calicheamicin, or crosslink DNA, like mitomycin. It is clear that DNA binding alone cannot explain the mechanism of action of DNA intercalators and other DNA-binding agents, because the equilibrium constants $\left(K_{\mathrm{d}}\right)$ for rather nonspecific binding of these compounds to DNA are often much larger than MIC values. The biological activity of DNA-binding compounds in many cases must rely on inhibition of a particular mechanism, likely one involving DNA interaction with a specific DNA-binding protein. The current work in conjunction with other recent studies from our group argues that doxorubicin and other anthracyclines as well as aloeemodin inhibit mycobacterial growth by inhibiting DnaG. Historically, anthracyclines have not been considered as leads as first-line anti-TB drugs owing to their toxicity and suppression of the immune system. Nevertheless, an anthracycline analog or its formulation with less toxicity against human cells, but still potent as a DnaG inhibitor, could be potentially useful as an antibacterial, especially against strains resistant to conventional anti-TB therapy. For example, the anthracycline aclacinomycin has long been known to be much less toxic than doxorubicin. ${ }^{28}$ The recent development of anticancer derivatives of doxorubicin with lower toxicity has been promising. Peptide-doxorubicin conjugates, such as AEZS-108, demonstrate higher anticancer selectivity and far lower toxicity against noncancer cells than doxorubicin. ${ }^{29-32}$ Analogous antibacterial targeting strategies for discovery of an antracycline analog could be envisioned. 4'-Iodo-4'deoxydoxorubicin, methoxymorpholinyl doxorubicin and 3'-azido doxorubicin are other examples of doxorubicin analogs with improved toxicity profiles. ${ }^{33-35}$ Liposomal formulations of doxorubicin have shown lower cardiac toxicity than doxorubicin. In summary, this study showcases DnaG as a potential target for future investigations with DNA intercalators and other inhibitory compounds.

\section{CONFLICT OF INTEREST}

The authors declare no conflict of interest.

\section{ACKNOWLEDGEMENTS}

This work was supported by startup funds from the College of Pharmacy at the University of Kentucky (to SG-T and OVT) and by a grant from the Israel Science Foundation (ISF, grant 58/10 to MF). We thank Dr Caixia Hou for helping with purification of $M t b$ DnaG.

1 Green, K. D. \& Garneau-Tsodikova, S. Resistance in tuberculosis: what do we know and where can we go? Frontiers Microbiol. 4, 208 (2013).

2 Wong, E. B., Cohen, K. A. \& Bishai, W. R. Rising to the challenge: new therapies for tuberculosis. Trends Microbiol. 21, 493-501 (2013).

3 Klann, A. G., Belanger, A. E., Abanes-De Mello, A., Lee, J. Y. \& Hatfull, G. F. Characterization of the dnaG locus in Mycobacterium smegmatis reveals linkage of DNA replication and cell division. J. Bacteriol. 180, 65-72 (1998).

4 Gefter, M. L., Hirota, Y., Kornberg, T., Wechsler, J. A. \& Barnoux, C. Analysis of DNA polymerases II and 3 in mutants of Escherichia coli thermosensitive for DNA synthesis. Proc. Natl Acad. Sci. USA 68, 3150-3153 (1971). 
5 van der Ende, A., Baker, T. A., Ogawa, T. \& Kornberg, A. Initiation of enzymatic replication at the origin of the Escherichia coli chromosome: primase as the sole priming enzyme. Proc. Natl Acad. Sci. USA 82, 3954-3958 (1985).

6 Karamata, D. \& Gross, J. D. Isolation and genetic analysis of temperature-sensitive mutants of $B$. subtilis defective in DNA synthesis. Molec. Gen. Genet. 108, 277-287 (1970)

7 Costa, A., Hood, I. V. \& Berger, J. M. Mechanisms for initiating cellular DNA replication. Ann. Rev. Biochem. 82, 25-54 (2013).

8 Keck, J. L., Roche, D. D., Lynch, A. S. \& Berger, J. M. Structure of the RNA polymerase domain of E. coli primase. Science 287, 2482-2486 (2000).

9 Biswas, T., Resto-Roldan, E., Sawyer, S. K., Artsimovitch, I. \& Tsodikov, O. V. A novel non-radioactive primase-pyrophosphatase activity assay and its application to the discovery of inhibitors of Mycobacterium tuberculosis primase DnaG. Nucl. Acids Res. 41, e56 (2013).

10 Biswas, T., Green, K. D., Garneau-Tsodikova, S. \& Tsodikov, O. V. Discovery of inhibitors of Bacillus anthracis primase DnaG. Biochemistry. 52, 6905-6910 (2013).

11 Patel, S., Sprung, A. U., Keller, B. A., Heaton, V. J. \& Fisher, L. M. Identification of yeast DNA topoisomerase II mutants resistant to the antitumor drug doxorubicin: implications for the mechanisms of doxorubicin action and cytotoxicity. Mol. Pharmacol. 52, 658-666 (1997).

12 Ward, D. C., Reich, E. \& Goldberg, I. H. Base specificity in the interaction of polynucleotides with antibiotic drugs. Science 149, 1259-1263 (1965).

13 Glaser, B. T. et al. A high-throughput fluorescence polarization assay for inhibitors of gyrase B. J. Biomol. Screen 16, 230-238 (2011).

14 Morlock, G. P., Metchock, B., Sikes, D., Crawford, J. T. \& Cooksey, R. C. ethA, inhA, and katG loci of ethionamide-resistant clinical Mycobacterium tuberculosis isolates. Antimicrob. Agents. Chemother. 47, 3799-3805 (2003).

15 Pecere, T. et al. Aloe-emodin is a new type of anticancer agent with selective activity against neuroectodermal tumors. Cancer Res. 60, 2800-2804 (2000).

16 Floridi, A. et al. Growth inhibition by rhein and lonidamine of human glioma cells in vitro. Anticancer Res. 10, 1633-1636 (1990).

17 Kuron, A. et al. Evaluation of DNA primase DnaG as a potential target for antibiotics. Antimicrob. Agents Chemother. 58, 1699-1706 (2014).

18 Smolarz, H. D., Swatko-Ossor, M., Ginalska, G. \& Medynska, E. Antimycobacterial effect of extract and its components from Rheum rhaponticum. J. AOAC Int. 96, 155-160 (2013).

19 Etienne, G. et al. The impact of the absence of glycopeptidolipids on the ultrastructure, cell surface and cell wall properties, and phagocytosis of Mycobacterium smegmatis. Microbiology 148(Pt 10), 3089-3100 (2002).

20 Piddock, L. J., Williams, K. J. \& Ricci, V. Accumulation of rifampicin by Mycobacterium aurum, Mycobacterium smegmatis and Mycobacterium tuberculosis. J. Antimicrob. Chemother. 45, 159-165 (2000).
21 Stephan, J., Mailaender, C., Etienne, G., Daffe, M. \& Niederweis, M. Multidrug resistance of a porin deletion mutant of Mycobacterium smegmatis. Antimicrob. Agents Chemother. 48, 4163-4170 (2004).

22 Yadav, A., Bhardwaj, R. \& Sharma, R. A. Phytochemical screening and antimicrobial activity of anthraquinones isolated from different parts of Cassia nodosa. Res. J. Med. Plant 7, 150-157 (2013).

23 Tegos, G., Stermitz, F. R., Lomovskaya, O. \& Lewis, K. Multidrug pump inhibitors uncover remarkable activity of plant antimicrobials. Antimicrob. Agents Chemother. 46, 3133-3141 (2002).

24 Duan, F. et al. Haloemodin as novel antibacterial agent inhibiting DNA gyrase and bacterial topoisomerase I. J. Med. Chem. 57, 3707-3714 (2014).

25 Taylor, J. A., Mitchenall, L. A., Rejzek, M., Field, R. A. \& Maxwell, A. Application of a novel microtitre plate-based assay for the discovery of new inhibitors of DNA gyrase and DNA topoisomerase VI. PLoS One 8, e58010 (2013).

26 Wallace, R. J. Jr. et al. Activities of ciprofloxacin and ofloxacin against rapidly growing mycobacteria with demonstration of acquired resistance following single-drug therapy. Antimicrob. Agents Chemother. 34, 65-70 (1990).

27 Ruiz-Serrano, M. J. et al. In vitro activities of six fluoroquinolones against 250 clinical isolates of Mycobacterium tuberculosis susceptible or resistant to first-line antituberculosis drugs. Antimicrob. Agents. Chemother. 44, 2567-2568 (2000).

28 Mathe, G. et al. Preliminary results of a phase II trial of aclacinomycin in acute leukaemia and lymphosarcoma. An oncostatic anthracyclin that is rarely cardiotoxic and induces no alopecia. Cancer Chemother. Pharmacol. 1, 259-262 (1978).

29 Soudy, R., Chen, C. \& Kaur, K. Novel peptide-doxorubucin conjugates for targeting breast cancer cells including the multidrug resistant cells. J. Med. Chem. 56, 7564-7573 (2013).

30 Grundker, C., Ernst, J., Reutter, M. D., Ghadimi, B. M. \& Emons, G. Effective targeted chemotherapy using AEZS-108 (AN-152) for LHRH receptor-positive pancreatic cancers. Oncol. Rep. 26, 629-635 (2011).

31 Emons, G., Sindermann, H., Engel, J., Schally, A. V. \& Grundker, C. Luteinizing hormone-releasing hormone receptor-targeted chemotherapy using AN-152. Neuroendocrinology 90, 15-18 (2009).

32 Engel, J. B., Schally, A. V., Buchholz, S., Seitz, S., Emons, G. \& Ortmann, O. Targeted chemotherapy of endometrial, ovarian and breast cancers with cytotoxic analogs of luteinizing hormone-releasing hormone (LHRH). Arch. Gynecol. Obstet. 286, 437-442 (2012)

33 Sessa, C. et al. Phase I clinical and pharmacological study of oral methoxymorpholinyl doxorubicin (PNU 152243). Cancer Chemother. Pharmacol. 44, 403-410 (1999).

34 Danesi, R. et al. Cardiac toxicity and antitumor activity of 4'-deoxy-4'-iodo-doxorubicinol. Cancer Chemother. Pharmacol. 26, 403-408 (1990).

$35 \mathrm{Yu}, \mathrm{S}$. et al. Synthesis and biological activities of a 3'-azido analogue of doxorubicin against drug-resistant cancer cells. Int. J. Mol. Sci. 13, 3671-3684 (2012). 